\title{
A new global search for the ground state structure of small cluster: application to $S_{6}$
}

\author{
Ming-Bang $\mathrm{Lv}^{a}$, Yan Cheng ${ }^{a, *}, \mathrm{Yi} \mathrm{Zhao}^{b}$, and $\mathrm{Min} \mathrm{Fu}^{a}$ \\ ${ }^{a}$ College of Physical Science and Technology, Sichuan University, Chengdu 610064, \\ China \\ ${ }^{b}$ Key Laboratory of Nuclear Fuel and Material, Nuclear Power Institute of China, \\ Chengdu 610041, China
}

Received 25 July 2012; Accepted (in revised version) 21 September 2012

Published Online 30 August 2013

\begin{abstract}
Initial configuration plays an important role in molecular dynamics (MD) simulation. Based on $a b$ initio MD simulation used widely, we here first introduce a global search method for the ground state structure of clusters. We think that during a simulation, each type of atoms in cluster should be in equivalent sites when we choose the initial configuration. Thus, our proposal is an improvement in eliminating the "unequal" in traditional annealing technology, and the results should be more convinced. Moreover, our method can make us obtain all possible structures of clusters, and also get some new structures, which are left out by previous studies. As an illustrative application, we here examine the $S_{6}$ cluster and found many structures (e.g. $\mathrm{D}_{3 d}, \mathrm{C}_{2 v}, \mathrm{D}_{3 h}, \mathrm{C}_{s}$ and $\mathrm{C}_{1}$ ). The geometrical parameters and vibrational frequencies are in agreement with experimental values.
\end{abstract}

PACS: 31.15.Ew, 31.15Qg, 36.40.-c

Key words: sulfur cluster, Langevin molecular dynamics, density functional theory

\section{Introduction}

Clusters exhibit distinct properties from atoms, molecules and solid states. It provides the possibility of understanding the transitions from atoms, molecules to crystalline solids. Among all the properties of clusters, the ground state structure is one of the most important properties. For very small clusters, one can create all conceivable structures and examine the total energy of each structure. However, once cluster size exceeds half a dozen atoms or so, it becomes nearly impossible to build all topologically distinct structures. Fortunately, computer simulations can help people to overcome this obstacle [1].

\footnotetext{
*Corresponding author. Email addresses: ycheng@scu.edu.cn (Y. Cheng)
} 
As is known that, Monte Carlo (MC) and molecular dynamics (MD) simulations are the two useful numerical technologies. Compared with the MC simulation, the traditional Newtonian MD simulation is much more efficient when energy surface is smooth, however, it is likely to be affected by the existence of local minima in complex energy surface. In 1986, Biswas and Hammann [2] proposed a procedure which combined some advantages of the MC technology with MD technology. They implemented a Langevin MD simulation for simulated annealing. Up to now, the Langevin MD simulations are widely applied to investigate the ground state structures of clusters [3-7].

Recently, we have investigated the ground state structure and optical properties of $S_{n}$ and $C_{n}(1<n<10)[6,7]$ by using a finite-difference pseudopotential density-functional theory method in real space and Langevin MD annealing technique through PARASEC code [8-10]. In general one can easily obtain the so-called ground state structures of clusters during a Langevin MD simulation. In fact, the so-called ground state of clusters is not a real ground state structure, but a metastable structure, because it depends on the chose of initial configuration of clusters.

Thus, we here place special emphasis on the initial configuration of cluster when an MD simulation is made. The initial configuration is related to the sample regions of phase space. In a traditional computer simulation, it is usually randomly constructed, which leads to the "unequal" atomic coordinates and then different force and potential energy. In other words, random coordinates let all atoms be in different surroundings. Moreover, many clusters, especially in large size, have complex energy surfaces with a number of candidate local energy minima and high-energy barriers, which trap the simulation in one of the numerous metastable configurations. Thus, the probability that a random initial structure leads to a global minima is very low. Due to these reasons, we here introduce a new global search for the ground state structure of clusters. We propose that each type of atoms in cluster should be in equivalent site. For instance, each type of atoms can be evenly distributed on a circle or on a round ball. To get the next initial configuration, we can only change the bond length $\mathrm{r}$ between two nearest atoms to $r+\Delta r$, where $\Delta r$ is defined as configuration step.

As an illustrative application, we make an investigation on the ground structure of $\mathrm{S}_{6}$ cluster. Our choice is motivated by several factors. Sulfur has the largest number of allotropic forms of any other elements in the period table [11,12], and it is unique in the propensity to catenation. The most interesting aspect is that many of the larger clusters $S_{n}$ $(n>5)[12,13]$ occur as monocyclic rings. Moreover, we are familiar with sulfur clusters, especially for $S_{6}$ [6]. Extensive studies of sulfur clusters have been performed [14-21], e.g. $\mathrm{S}_{5}-\mathrm{S}_{8}$ rings by Cioslowski et al. [14], $\mathrm{S}_{2}-\mathrm{S}_{11}$ by Chen et al. [15], $\mathrm{S}_{2}-\mathrm{S}_{12}$ by Millefiori and Alparone [16], $\mathrm{S}_{6}-\mathrm{S}_{16}$ by Peter [17], $\mathrm{S}_{2}-\mathrm{S}_{20}$ by Ludwig et al. [18], $\mathrm{S}_{2}-\mathrm{S}_{18}$ by Jones and Ballone [19], $S_{2}-S_{10}$ by Steudel et al. [20], and $S_{6}$ by Wong et al. [21].

The rest of the paper is organized as follows. The theoretical method and the calculation details are given in Section 2. Some results and discussion are presented in Section 3. Finally, the summary of our main results are given in Section 4 . 


\section{Theoretical method and calculation details}

The structure of $S_{6}$ cluster is investigated via simulated annealing method based on Langevin molecular dynamics, which is described in details in Refs. [3-5, 8-10]. Here we only make a brief introduction for this theory.

The ionic positions, $R_{j}$, in the form of dynamics, evolve according to

$$
M_{j} \ddot{R}_{j}=F\left(R_{j}\right)-\gamma M_{j} \dot{R}_{j}+G_{j},
$$

where $M_{j}$ is the ionic masses, $F\left(R_{j}\right)$ is the interatomic force on the $j$-th particle, ${ }_{\gamma} M_{j} R_{j}$ is the dissipation force and $G_{j}$ is the fluctuation force. The dissipative forces are defined by the friction coefficient $\gamma$. The fluctuation forces are defined by random Gaussian variables, Gj, with a white noise spectrum. In order to obtain the realistic interatomic forces, $F$, which is the most demanding issue in implementing Langevin dynamics, we have used the density-functional theory and pseudopotentials. The total ground is expressed as follows within the density functional theory

$$
E+_{\text {total }}=T(\rho)+E_{e-i}\left(R_{\alpha}, \rho\right)+E_{h}(\rho)+E_{x c}(\rho)+E_{i-i}\left(R_{\alpha}\right),
$$

where $T(\rho)$ is the kinetic energy, $E_{e-i}$ is ionic potential energy, $E_{h}$ is the Hartree potential energy, $E_{x c}$ is the exchange-correlation energy, $E_{i-i}$ is the inter-ionic core interaction energy, $\rho(r)=\sum_{n}\left|\Psi_{n}(r)\right|^{2}$ is the ground state valence charge density where the sum is over occupied states, and $\Psi_{n}(r)$ is the ground state wave functions. The force, $F_{a}^{\alpha}$, on an atom located at $R_{a}$ in the $\alpha$ direction for a finite system is obtained using the HellmannFeynman theorem

$$
F_{a}^{\alpha}=-\frac{e E_{t o t a l}}{d R_{a}^{\alpha}}=-\frac{E_{e-i}}{R_{a}^{\alpha}}=-\frac{E_{i-i}}{R_{a}^{\alpha}}
$$

The inter-ionic core interaction is simply the point-charge-point-charge interaction under the frozen core approximation. It is the direct pair summation of Coulomb interactions for an isolated system, and an Ewald summation for a periodic system. A complicating issue is that the ionic term is described by a non-local ionic pesudopotential.

The exchange-correlation potential term is approximated by the Ceperley-Alder functional [22,23]. The Troullier-Martins nonlocal pseudopotentials [24] in Kleinman-Bylander form [25] are used. The core cut-off radii adopted are all 1.70 a.u. [6, 7] for S 3s, 3p, and $3 d$ electronic states in constructing the Troullier-Martins scheme pseudopotentials. With respect to computing the quantum forces, the cluster in question is placed in a spherical domain. Outside of this domain, the wave functions are required to vanish. The radius of the sphere is such that the outmost atom is $7 \mathrm{a}$.u. from the boundary. The grid spacing is 0.5 a.u. As a rough estimate, one can compare this grid spacing with a plane wave cutoff of $(\pi / h)^{2}$ or about 40 Ry for $h=0.5$ a.u. The initial temperature of this simulation is taken to be $1000 \mathrm{~K}$ (above the boiling point); the final temperature is taken to be $100 \mathrm{~K}$ (below the fusion point). The annealing schedule lowered the temperature $100 \mathrm{~K}$ each 50 time-steps. The time-step is taken to be 250 a.u. The friction coefficient in the Langevin 
equation is taken to be 0.0006 a.u. After reaching the temperature of $100 \mathrm{~K}$, the cluster is quenched to $0 \mathrm{~K}$.

The ground state structure of $\mathrm{S}_{6}$ cluster is found through a direct minimization by a steepest descent procedure. Considering affects of the initial configuration of clusters, we propose that each type of atoms should be in equivalent sites when we build the initial configuration of clusters. Here we choose an octahedron, with each $S$ atom situated at the vertex equally, which make sure six atoms distribute evenly in a sphere. By changing the radius of the sphere with the configuration step $\Delta r$, we can obtain many initial configurations for our dynamics simulations. Of course, an appropriate range of bond length is necessary. If the atoms are too far apart, they will exhibit Brownian motion and may not form a stable cluster as the simulation proceeds. If the atoms are too close together, they may form a metastable cluster from which the ground state may be kinetically inaccessible. Typically, a random placement of the atoms must reside within 1.05 and 1.30 times the dimer bond length. If the initial atomic configuration cannot form a stable cluster, two methods can be useful in dealing with such problems. One method is that, the energy per atom may be checked in the first 50 time-steps to confirm if the difference of the energy from that of $S_{2}$ dimer is no more or less than $4 \mathrm{eV}$. When the difference is much greater, one must reform the initial configuration. The other method is that, the number of the time-steps may be taken less than $50[6,7]$.

Along with the shaping of stable structure in simulations, the binding energies $E_{b i n d}$ and the kinetic energy $E_{k i n}$ finally converge to a constant, though both of them remain slightly fluctuant. To obtain more accurate results, we perform further structural optimization by high-level $a b$ initio molecular orbital calculations with the final structure from simulated annealing. Raghavachari et al. [26] showed that the inclusion of $d$-type functions in the basis set was found to be extremely important in obtaining reliable geometries, the bond lengths being too long if they were excluded. Also, previous study $[14,27]$ showed that electron correlation effects play an important role in conformational analysis of sulfur clusters. Thus, the further geometry optimization and vibrational analysis here are perform at B3LYP/6-311G ( $3 d f, 3 p d)$ level theory.

\section{Results and discussion}

We take the initial geometry of $\mathrm{S}_{6}$ cluster as an octahedron, and change the bond length $\mathrm{r}$ of initial structure from 3.2 a.u to 5.0 a.u with $\Delta r=0.2$ a.u. With the initial configuration of each bond length, the anneal procedures are performed, and then ten final structures are obtained.

In Fig. 1, as an example, we illustrate the evolution of binding energies $E_{b i n d}$ and the kinetic energy $E_{k i n}$ during a Langevin simulation with $r=4.2$ a.u. The gradually reduced fluctuation indicates that the exploring tends to more stable structures, and the ultimate configuration eventually takes shape. The evolution of structure during Langevin simulations are illustrated in Fig. 2(a) and Fig. 2(b) corresponding to the initial bond length 


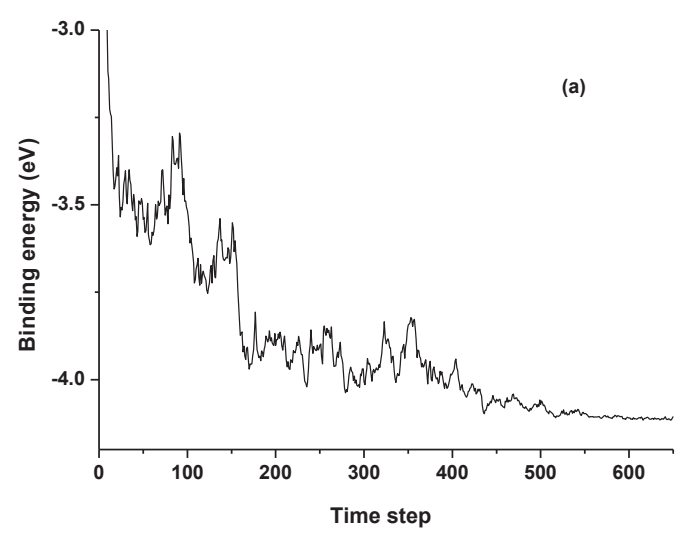

(a)

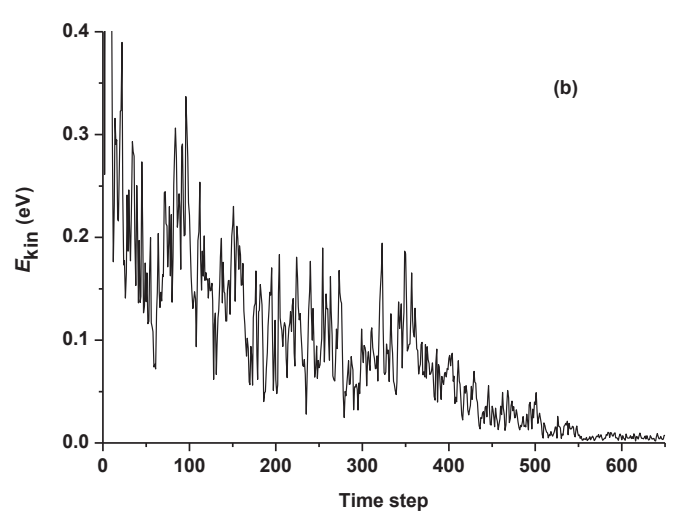

(b)

Figure 1: Binding energy (a) and $E_{k i n}$ (b) of $\mathrm{S}_{6}$ cluster during a Langevin simulation with $r=4.2$ a.u. The initial temperature is $1000 \mathrm{~K}$ and the final temperature is $100 \mathrm{~K}$. The time step is $250 \mathrm{a}$.u. (about $6.04 \mathrm{fs}$ ).

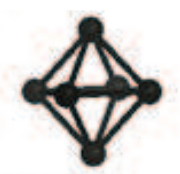

(a) $\mathrm{t}=0 \mathrm{fs}$

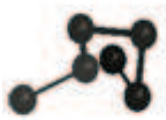

(d) $t=362.4 \mathrm{fs}$

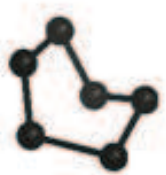

(g) $\mathrm{t}=815.4 \mathrm{fs}$ (D)

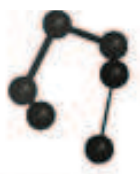

(b) $\mathrm{t}=90.6 \mathrm{fs}$

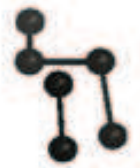

(e) $t=543.6 \mathrm{fs}$

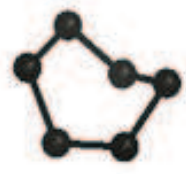

(h) $\mathrm{t}=906.0 \mathrm{fs}$

$\left(\mathrm{C}_{2}\right)$

(a)

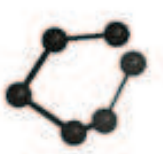

(c) $t=181.2 \mathrm{fs}$

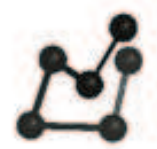

(f) $\mathrm{t}=724.8 \mathrm{fs}$

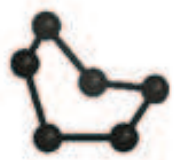

(i) $\mathrm{t}=1087.2 \mathrm{fs}$

$\left(\mathrm{C}_{2 \mathrm{~V}}\right)$

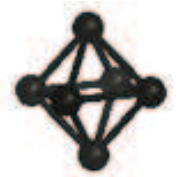

(a) $t=0 \mathrm{fs}$

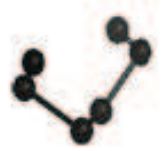

(d) $t=1087.2 \mathrm{fs}$

(e) $\mathrm{t}=1630.8 \mathrm{fs}$
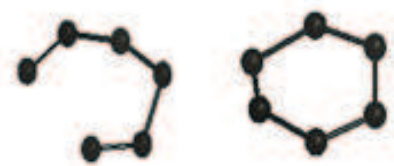

(g) $t=3080.4 \mathrm{fs}$

(h) $t=3261.6 \mathrm{fs}$

fs (i)

$t=3442.3 \mathrm{fs}$

(b)

Figure 2: The geometries of $\mathrm{S}_{6}$ cluster of at various time step during a Langevin simulation with $r=5.0$ a.u, 4.2 a.u. The most common structures possessing $D_{3 d}$ and $C_{2 v}$ symmetry are achieved.

of 5.0 a.u and $4.2 \mathrm{a} . \mathrm{u}$, respectively. The most common structures possessing $\mathrm{D}_{3 d}$ and $\mathrm{C}_{2 v}$ symmetry are achieved with the above bond length of the initial structures.

We obtain five distinct isomers (two $D_{3 d}, C_{s}, C_{1}$, three $C_{2 v}$ and one $D_{3 h}$ ) as the last 


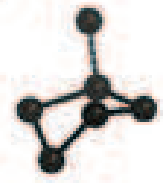

(a) 3.2 a.u

$\mathrm{C}_{1}$

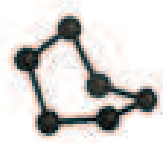

(d) 3.8 a.u

$\mathrm{C}_{2 \mathrm{v}}$

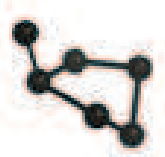

(g) 4.4 a.u

$\mathrm{C}_{1}$

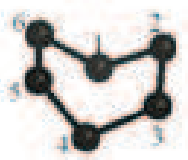

(j) 5.0 a.u

$\mathrm{C}_{2 v}$

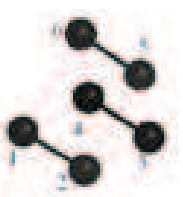

(b) $3.4 \mathrm{au}$

$\mathrm{D}_{3 \mathrm{k}}$

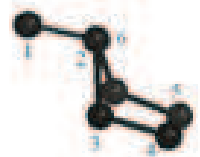

$\mathrm{C}_{2 \mathrm{v}}$

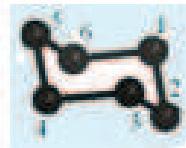

(e) $4.0 \mathrm{a} . \mathrm{u}$

(f) $4.2 \mathrm{au}$

$\mathrm{C}_{1}$

$\mathrm{D}_{3 \mathrm{c}}$
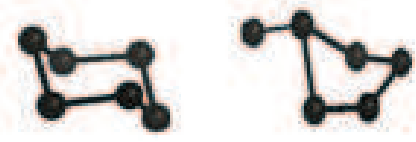

(h) $4.6 \mathrm{au}$

(i) 4.8 a.u

$\mathrm{D}_{3 \mathrm{~d}}$

$\mathrm{C}_{\mathrm{B}}$

Figure 3: The obtained final structures during Langevin simulations and the corresponding initial bond lengths.

results of the simulated anneal procedures. Owing to space constraints, we do not show all anneal procedures but the eventual results. The bond length of the initial structures and the corresponding simulation results are illustrated in Fig. 3. Interestingly, the final configurations in Fig. 3 may occur in another simulation process. For instance, the structure of $C_{s}$ symmetry is detected in Fig. 2(b). The twist $D_{2}$ and $C_{2}$ structures are also found in Fig. 2(a).

The further geometry optimization at B3LYP/6-311G ( $3 d f, 3 p d)$ level theory are performed with the final structures of anneal procedure as its initial configuration. Inci- 
Table 1: Geometrical parameters and relative energy $(\mathrm{kcal} / \mathrm{mol})$ for $\mathrm{S}_{6}$ cluster calculated at the B3LYP/6$311 \mathrm{G}(3 d f, 3 p d)$ level.

\begin{tabular}{|c|c|c|c|c|c|c|}
\hline \multirow{2}{*}{ Symmetries } & & \multicolumn{4}{|c|}{ Geometrical parameters } & \multirow{2}{*}{$\begin{array}{c}\text { Relative } \\
\text { energy }\end{array}$} \\
\hline & & \multicolumn{2}{|c|}{ Other theoretical studies } & This work & Expt.[22,23] & \\
\hline $\begin{array}{c}\mathrm{S}_{6}(\mathrm{a}) \\
r=4.2 \mathrm{a} . \mathrm{u}\end{array}$ & $\mathrm{D}_{3 d}$ & $\begin{array}{l}\text { Hohl et al. }[24] \\
r_{12}=2.11 \\
\alpha_{123}=102 \\
\beta_{1234}=75\end{array}$ & $\begin{array}{l}\text { Raghavachari et al. [20] } \\
r_{12}=2.06 \\
\alpha_{123}=102.5 \\
\beta_{1234}=73.9\end{array}$ & $\begin{array}{l}r_{12}=2.08 \\
\alpha_{123}=102.9 \\
\beta_{1234}=73.2\end{array}$ & $\begin{array}{l}r_{12}=2.07 \\
\alpha_{123}=102.6 \\
\beta_{1234}=73.8\end{array}$ & 0.0 \\
\hline $\begin{array}{c}\mathrm{S}_{6}(\mathrm{~b}) \\
r=5.0 \mathrm{a} \cdot \mathrm{u}\end{array}$ & $\mathrm{C}_{2 v}$ & $\begin{array}{l}\text { Hohl et al. }[24] \\
r_{12}=2.07 \\
r_{12}=2.22 \\
\alpha_{612}=102 \\
\alpha_{123}=101 \\
\beta_{6123}=75 \\
\beta_{1234}=0\end{array}$ & $\begin{array}{l}\text { Raghavachari et al. [20] } \\
r_{12}=2.064 \\
r_{23}=2.160 \\
\alpha_{612}=104.8 \\
\alpha_{123}=103.6 \\
\beta_{6123}=71.6 \\
\beta_{1234}=0.0\end{array}$ & $\begin{array}{l}r_{12}=2.044 \\
r_{23}=2.22 \\
\alpha_{612}=107.0 \\
\alpha_{123}=101.9 \\
\beta_{6123}=73.4 \\
\beta_{1234}=0.0\end{array}$ & & 12.24 \\
\hline $\begin{array}{c}\mathrm{S}_{6}(\mathrm{c}) \\
r=3.4 \mathrm{a} \cdot \mathrm{u}\end{array}$ & $\mathrm{D}_{3 b}$ & & $\begin{array}{l}\text { Wong et al. [13] } \\
r_{12}=1.90 \\
r_{14}=2.76 \\
\alpha_{123}=90.0 \\
\alpha_{612}=60.0 \\
\beta_{1234}=0.0 \\
\beta_{6123}=60.0\end{array}$ & $\begin{array}{l}r_{12}=1.89 \\
r_{14}=2.76 \\
\alpha_{123}=90.0 \\
\alpha_{612}=60.0 \\
\beta_{1234}=0.0 \\
\beta_{6123}=60.0\end{array}$ & & 14.43 \\
\hline $\begin{array}{c}\mathrm{S}_{6}(\mathrm{~d}) \\
r=4.0 \mathrm{a} . \mathrm{u}\end{array}$ & $\mathrm{C}_{S}$ & & $\begin{array}{l}\text { Wong et al. [13] } \\
r_{12}=1.929 \\
r_{23}=2.313 \\
r_{34}=1.98 \\
r_{45}=2.285 \\
\alpha_{123}=107.4 \\
\alpha_{345}=101.3 \\
\alpha_{326}=82.2 \\
\alpha_{234}=100.9 \\
\beta_{4562}=43.4 \\
\beta_{1234}=170.1 \\
\beta_{3456}=0.0\end{array}$ & $\begin{array}{l}r_{12}=1.917 \\
r_{23}=2.323 \\
r_{34}=1.97 \\
r_{45}=2.294 \\
\alpha_{123}=107.7 \\
\alpha_{345}=101.2 \\
\alpha_{326}=82.2 \\
\alpha_{234}=101.0 \\
\beta_{4562}=43.8 \\
\beta_{1234}=170.6 \\
\beta_{3456}=0.0\end{array}$ & & 23.66 \\
\hline
\end{tabular}

Denote: $r=$ bond length (A), $\alpha=$ bond angle (deg.), $\beta=$ dihedral angle (deg.). Atom numbering is given in the Fig. 3.

dentally, the same symmetry structures which are achieved from anneal procedure of different band length (e.g. 4.6 a.u, 4.2 a.u) have nearly the same geometrical parameters and frequencies. So, we only list four (two $C_{1}$ structures without symmetry are ignored) of the ten structures to compare with other results. In Table 1, we present the geometrical parameters and relative energies of $S_{6}$ cluster. The calculated harmonic vibrational frequencies, the available experimental data, and the corresponding zero-point energies of these structures are listed in Table 2.

From Tables 1 and 2, we can see that there exist four calculated isomers corresponding to initial bond length 4.2 a.u, 5.0 a.u, 3.4 a.u and 4.0 a.u, respectively, i.e. $S_{6}(a), S_{6}(b), S_{6}$ (c), and $S_{6}(d) . S_{6}(a)$ has a chair-shaped structure, which is essentially formed early after 
Table 2: Vibrational frequencies $\left(\mathrm{cm}^{-1}\right)$ and zero-point energies $(\mathrm{kcal} / \mathrm{mol})$ for $\mathrm{S}_{6}$ cluster, B3LYP/6-311G(3df, $3 p d)$.

\begin{tabular}{|c|c|c|c|c|c|}
\hline Molecule & Symmtry & Other theoretical studies & This work & Expt. & ZPE \\
\hline $\begin{array}{c}\mathrm{S}_{6}(\mathrm{a}) \\
r=4.2 \mathrm{a} . \mathrm{u}\end{array}$ & $\mathrm{D}_{3 d}$ & $\begin{array}{c}\text { Raghavachari et al. [20] } \\
159\left(\mathrm{e}_{u}\right) \\
214\left(\mathrm{e}_{g}\right) \\
259\left(\mathrm{a}_{1 g}\right) \\
325\left(\mathrm{a}_{g}\right) \\
439\left(\mathrm{a}_{1 u}\right) \\
447\left(\mathrm{e}_{u}\right) \\
457\left(\mathrm{e}_{g}\right) \\
457\left(\mathrm{a}_{1 g}\right)\end{array}$ & $\begin{array}{c}164\left(\mathrm{e}_{u}\right) \\
203\left(\mathrm{e}_{g}\right) \\
264\left(\mathrm{a}_{1 g}\right) \\
314\left(\mathrm{e}_{g}\right) \\
341\left(\mathrm{a}_{1 u}\right) \\
431\left(\mathrm{e}_{u}\right) \\
454\left(\mathrm{e}_{g}\right) \\
474\left(\mathrm{a}_{1 g}\right)\end{array}$ & $\begin{array}{c}180\left(\mathrm{e}_{u}\right) \\
203\left(\mathrm{e}_{g}\right) \\
265\left(\mathrm{a}_{1 g}\right) \\
312\left(\mathrm{e}_{g}\right) \\
390\left(\mathrm{a}_{1 u}\right) \\
451\left(\mathrm{e}_{u}\right) \\
462\left(\mathrm{e}_{g}\right) \\
477\left(\mathrm{a}_{1 g}\right)\end{array}$ & 5.57 \\
\hline $\begin{array}{c}\mathrm{S}_{6}(\mathrm{~b}) \\
r=5.0 \text { a.u }\end{array}$ & $\mathrm{C}_{2 v}$ & $\begin{array}{c}\text { Raghavachari et al. [20] } \\
38 \mathrm{i}\left(\mathrm{a}_{2}\right) \\
178\left(\mathrm{~b}_{2}\right) \\
204\left(\mathrm{a}_{1}\right) \\
220\left(\mathrm{a}_{2}\right) \\
240\left(\mathrm{a}_{1}\right) \\
316\left(\mathrm{~b}_{2}\right) \\
381\left(\mathrm{~b}_{1}\right) \\
390\left(\mathrm{a}_{1}\right) \\
467\left(\mathrm{a}_{2}\right) \\
456\left(\mathrm{~b}_{1}\right) \\
477\left(\mathrm{~b}_{2}\right) \\
481\left(\mathrm{a}_{1}\right)\end{array}$ & $\begin{array}{c}45\left(a_{2}\right) \\
183\left(b_{2}\right) \\
207\left(a_{1}\right) \\
207\left(a_{2}\right) \\
231\left(a_{1}\right) \\
311\left(b_{2}\right) \\
315\left(b_{1}\right) \\
374\left(a_{1}\right) \\
418\left(b_{1}\right) \\
430\left(a_{2}\right) \\
494\left(b_{2}\right) \\
503\left(a_{1}\right)\end{array}$ & & 5.32 \\
\hline $\begin{array}{c}\mathrm{S}_{6}(\mathrm{c}) \\
r=3.4 \text { a.u }\end{array}$ & $\mathrm{D}_{3 h}$ & $\begin{array}{c}\text { Wong et al. [13] } \\
82\left(\mathrm{e}^{\prime \prime}\right) \\
131\left(\mathrm{a}_{2}^{\prime \prime}\right) \\
192\left(\mathrm{e}^{\prime}\right) \\
245\left(\mathrm{a}_{1}^{\prime \prime}\right) \\
265\left(\mathrm{e}^{\prime}\right) \\
294\left(\mathrm{e}^{\prime \prime}\right) \\
682\left(\mathrm{e}^{\prime}\right) \\
728\left(\mathrm{a}_{1}^{\prime}\right)\end{array}$ & $\begin{array}{c}85\left(\mathrm{e}^{\prime \prime}\right) \\
130\left(\mathrm{a}_{2}{ }^{\prime \prime}\right) \\
190\left(\mathrm{e}^{\prime}\right) \\
245\left(\mathrm{a}_{1}{ }^{\prime \prime}\right) \\
265\left(\mathrm{a}_{1}{ }^{\prime}\right) \\
295\left(\mathrm{e}^{\prime \prime}\right) \\
682\left(\mathrm{e}^{\prime}\right) \\
730\left(\mathrm{a}_{1}{ }^{\prime}\right)\end{array}$ & & 5.53 \\
\hline $\begin{array}{c}\mathrm{S}_{6}(\mathrm{~d}) \\
r=4.0 \mathrm{a} . \mathrm{u}\end{array}$ & $\mathrm{C}_{s}$ & $\begin{array}{l}\text { Wong et al.[13] } \\
\text { 91( }\left(\alpha^{\prime \prime}\right) \\
91\left(\alpha^{\prime}\right) \\
124\left(a^{\prime \prime}\right) \\
183\left(a^{\prime}\right) \\
206\left(a^{\prime}\right) \\
\left.219\left(a^{\prime \prime}\right)\right) \\
287\left(a^{\prime}\right) \\
313\left(a^{\prime \prime}\right) \\
340\left(a^{\prime}\right) \\
560\left(a^{\prime}\right) \\
567\left(a^{\prime \prime}\right) \\
654\left(a^{\prime}\right)\end{array}$ & $\begin{array}{c}89\left(\alpha^{\prime \prime}\right) \\
94\left(\alpha^{\prime}\right) \\
129\left(a^{\prime \prime}\right) \\
177\left(a^{\prime}\right) \\
200\left(a^{\prime}\right) \\
217\left(a^{\prime \prime}\right) \\
274\left(a^{\prime}\right) \\
312\left(a^{\prime \prime}\right) \\
337\left(a^{\prime}\right) \\
565\left(a^{\prime}\right) \\
568\left(a^{\prime \prime}\right) \\
655\left(a^{\prime}\right)\end{array}$ & & 5.17 \\
\hline
\end{tabular}


about 180 steps (about $1087.2 \mathrm{fs}$ ). This structure has the $\mathrm{D}_{3 d}$ symmetry, corresponding to a global energy minimum. $\mathrm{S}_{6}(\mathrm{~b})$ has a boat-shaped $\mathrm{C}_{2 v}$ symmetry, which is formed after 540 steps (about $3442.3 \mathrm{fs}$ ). Before $C_{2 v}$ is essentially formed, we have found the $D_{2}-C_{2 v}$ interconversion, via intermediate $\mathrm{C}_{2}$, which is presented in Fig. 2. That also provides visual evidence for previous studies $[14,26,27]$ of such interconversion of sulfur cluster. $\mathrm{S}_{6}$ (c) has a $\mathrm{D}_{3 d}$ symmetry of trigonal prism. The prism structure is essentially a cluster of three $S_{2}$ molecules connected via a six-center $\pi^{*}-\pi^{*}-\pi^{*}$ interaction [21]. Although many of the large clusters $(n>5)$ occur as monocyclic rings, the special configuration might give us some inspirations about the way in which the larger clusters take shape. Finally, $\mathrm{S}_{6}(\mathrm{~d})$ has a semi-plane structure. Our calculations about geometrical parameters are in excellent agreement with the available experimental values and other results.

Raghavachari et al. [26] conducted the detailed frequency analysis based on ab initio calculations at the HF/3-21G* level, but their results are not accurate enough. He also claimed that such level of theory make calculated frequencies $10 \%-15 \%$ larger than the experimental values, and a uniform scaling factor 0.89 [26] were used to compare with the experimental data in their paper. For $\mathrm{S}_{6}(\mathrm{a})$, the calculated frequencies 341 (a1u) is slightly smaller than the experimental value 390 (a1u), while Raghavachari et al's value 439 (a1u) is a little overestimated. The vibrational frequencies of $S_{6}(b)$ reveals that $C_{2 v}$ structure is a local minimum instead of a transition state, due to the inexistence of imaginary frequencies, which is disagreement with published HF/3-21G* studies [26, 27]. Incidentally, the structures of local minima are characterized rigorously by the presence of all positive frequencies. To confirm our results, we also perform calculations at the HF/3-21G* level. As we expect, there appears an imaginary frequency $(-44)$ as a signal of transition state, underlining the importance of inclusion of electron effects in conformational analysis of sulfur clusters. The frequencies of $S_{6}$ (b) do not accord well with those of Raghavachari et al. Such disaccord may result from the omitting of electron effects and the uncertain uniform scaling factor. Calculated frequencies of $S_{6}(c)$ and $S_{6}(d)$ are in good agreement with previous calculations [21].

\section{Conclusions}

Initial configuration, which is closely linked to the final structure in annealing process, plays an important role in computer simulation. Based on ab initio MD simulation used widely, we here first introduce a global search method for the ground state structure of clusters. We think that during a simulation, each type of atoms in cluster should be in equivalent sites when we choose the initial configuration. Thus, our proposal is an improvement in eliminating the "unequal" in traditional annealing technology, and the results should be more convinced. Moreover, if we set the configuration step small enough, the search will definitely obtain all possible structures of clusters, and also get some new structures which are left out by previous studies. Compared with our global search, a random initial structure can only get "might" ground state, for clusters usually 
have complex energy surfaces with many candidate local energy minima. Our proposal is also recommended to be coupled with the genetic algorithm [31] of molecular geometry optimization.

Acknowledgments. The authors would like to thank the support by the National Natural Science Foundation of China under Grant Nos. 11174214 and 11204192, and the National Key Laboratory for Nuclear Fuel and Materials of Nuclear Power Institute of China under Grant No. STRFML-2012-06.

\section{References}

[1] J. R. Chelikowsky, N. Troullier, X. Jing, et al., Comput. Phys. Commun. 85 (1995) 325.

[2] R. Biswas and D. R. Hamann, Phys. Rev. B 34 (1986) 895.

[3] S. Ogut J. R. Chelikowsky, and S. G. Louie, Phys. Rev. Lett. 79 (1997) 1770.

[4] S. Li, M. M. G. Alemany, and J. R. Chelikowsky, Phys. Rev. B 71 (2005) 165433.

[5] X. Huang, A. Makmal, J. R. Chelikowsky, and L. Kronik, Phys. Rev. Lett. 94 (2005) 236801.

[6] Y. L Bai, X. R Chen, X. D Yang, and X. L Zhou, J. Phys. B: At. Mol. Opt. Phys. 36 (2003) 4511; X. R. Chen, Y. L Bai, J. Zhu, and Q. Q. Gou, Phys. Lett. A 316 (2003) 413.

[7] J. Q. Lan, X. R. Chen, Y. L. Bai, and J. Zhu, Physica B 405 (2010) 148.

[8] J. R. Chelikowsky, N. Troullier, and Y. Saad, Phys. Rev. Lett. 72 (1994) 1240.

[9] C. Troparevsky and J. R. Chelikowsky, J. Chem. Phys. 114 (2001) 943

[10] M. M. G. Alemany, M. Jain, L. Kronik, and J. R. Chelikowsky, Phys. Rev. B 69 (2004) 075101.

[11] R. Steudel, Topic in Current Chem. 102 (1982) 149.

[12] R. Steudel, in: Studies in Inorganic Chemistry 5, edited by A. Muller and B. Krebs (Elsevier,Amsterdam,1984)

[13] J. Donohue, The Structures of the Elements (Wiley, New York, 1974).

[14] J. Cioslowski, A. Szarecka, and D. Moncrieff, J. Phys. Chem. A 105 (2001) 501.

[15] M. D. Chen, M. L. Liu, H. B. Luo, Q. E. Zhang, and C. T. Au, J. Mol. Struct. (Theochem.) 548 (2001) 133.

[16] S. Millefiori and A. Alparone, J. Phys. Chem. A 105 (2001) 9489.

[17] L. Peter, Phosphorus. Sulfur Silicon Relat. Elem. 168 (2001) 287.

[18] R. Ludwig, J. Behler, B. Klink, and F. Weinhold, Angew. Chem. Int. Ed. 41 (2002) 3199.

[19] R. O. Jones and P. Ballone, J. Chem. Phys. 118 (2003) 9257.

[20] R. Steudel, Y. Steudel, and M. W. Wong, Top. Curr. Chem. 230 (2003) 117.

[21] M. W. Wong, Y. Steudel, and R. Steudel, J. Chem. Phys. 121 (2004) 5899.

[22] D. M. Ceperley and B .J. Alder, Phys. Rev. Lett. 45 (1980) 566.

[23] J. P. Perdew and A. Zunger, Phys. Rev. B 23 (1981) 5048.

[24] N. Troullier and J. L. Martins, Phys. Rev. B 43 (1991) 1993.

[25] L. Kleinman and D. M. Bylander, Phys. Rev. Lett. 48 (1982) 1425.

[26] K. Raghavachari, C. M. Rohlfing, and J. S. Binkley, J. Chem. Phys. 93 (1990) 5862.

[27] R. J. Suontamo, R. S. Laitinen, and T. A. Pakkanen, Acta Chem. Scand. 45 (1991) 687.

[28] S. Millefiori and A. Alparone, J. Phys. Chem. 105 (2001) 9489.

[29] S. J. Rettig and J. Trotter, Acta Cryst. C 43 (1987) 2260.

[30] D. Hohl, R. O. Jones, R. Car, and M. Parrinello, J. Chem. Phys. 89 (1988) 6823.

[31] D. M. Deaven and K. M. Ho, Phys. Rev. Lett. 75 (1995) 288. 